\title{
Characterization of Ebinur Lake Virus and Its Human Seroprevalence at the China-Kazakhstan Border
}

\author{
Han Xia't, Ran Liu'ti, Lu Zhao ${ }^{1,3+}$, Xiang Sun ${ }^{4}$, Zhong Zheng ${ }^{4}$, Evans Atoni ${ }^{1,3}$, \\ Xiaomin $\mathrm{Hu}^{1}$, Bo Zhang ${ }^{1}$, Guilin Zhang ${ }^{4 *}$ and Zhiming Yuan ${ }^{1 \star}$ \\ 1 Key Laboratory of Special Pathogens and Biosafety, Wuhan Institute of Virology, Chinese Academy of Sciences, Wuhan, \\ China, ${ }^{2}$ Illumina (China), Beijing, China, ${ }^{3}$ University of Chinese Academy of Sciences, Beijing, China, ${ }^{4}$ Center for Disease \\ Control and Prevention of Xinjiang Uygur Autonomous Region, Urumqi, China
}

\section{OPEN ACCESS}

Edited by:

Akio Adachi,

Kansai Medical University, Japan

Reviewed by:

Xiaohong Shi

University of Glasgow,

United Kingdom

Robert Tesh,

The University of Texas Medical

Branch, United States

*Correspondence:

Guilin Zhang

xjglzhang@126.com

Zhiming Yuan

yzm@wh.iov.cn

${ }^{\dagger}$ These authors have contributed equally to this work

Specialty section:

This article was submitted to

Virology,

a section of the journal

Frontiers in Microbiology

Received: 08 October 2019 Accepted: 23 December 2019

Published: 30 January 2020

Citation:

Xia H, Liu R, Zhao L, Sun X, Zheng Z, Atoni $E$, Hu X, Zhang $B$,

Zhang $G$ and Yuan Z (2020)

Characterization of Ebinur Lake Virus and Its Human Seroprevalence at the China-Kazakhstan Border.

Front. Microbiol. 10:3111. doi: 10.3389/fmicb.2019.03111
In recent years, rapidly increasing trade and travel across the China-Kazakhstan border has increased the potential risk of the introduction and exportation of vectors and their related diseases. The Ebinur Lake Nature Reserve is located in Xinjiang Uygur Autonomous Region, near the China-Kazakhstan border, with a suitable ecosystem for mosquito breeding. In our previous work, a novel Orthobunyavirus species named Ebinur Lake virus (EBIV) was isolated in the reserve. To gain insights into the potential risk of EBIV in this region, we conducted a study that aimed to clearly outline EBIV's biological characteristics and its human seroprevalence in this region. Phylogenetically, the analysis of all three segments of EBIV demonstrated that it belongs to the genus Orthobunyavirus, which is clustered in the Bunyamwera serogroup. EBIV replicated efficiently and caused cytopathic effects (CPEs) in vertebrate cells. The survival rates of the EBIV-challenged mice were 0 and $20 \%$ when inoculated with viral concentrations $\geq 10^{4}$ or $10^{2}$ plaque-forming units, respectively. For EBIV-infected mice, internal bleeding and pathological changes were observed. In addition, the overall immunoglobulin M (IgM) antibody [1:4 by immunofluorescence assay (IFA)], immunoglobulin $G$ (IgG) antibody (1:10 by IFA), and neutralizing antibody [90\% plaque reduction neutralization test (PRNT)] prevalence was 8.05, 12.3, and 0.95\%, respectively, in the studied residents. In summary, EBIV is a new member of the Bunyamwera serogroup and is able to competently infect cells derived from mosquitoes, rodents, monkeys, or humans. Furthermore, EBIV caused severe disease and even death in challenged Kunming mice, and the antibodies against EBIV have been detected in local residents, indicating that the virus is a potential animal or human pathogen.

Keywords: Xinjiang, Ebinur Lake virus, Culex modestus, mosquito, Orthobunyavirus

\section{INTRODUCTION}

Mosquito-borne viruses are naturally maintained in sylvatic and urban cycles of mosquitoes, as vectors, and susceptible vertebrate hosts, as reservoirs or dead-end hosts (Hollidge et al., 2010). However, studies that focus on neglected mosquito-borne viruses are still inadequate since much attention and priority has been given to classical arboviruses that have previously caused outbreaks. 
Ebinur Lake, the largest saltwater lake in western Xinjiang Uygur Autonomous Region, China, is located within the Ebinur Lake Wetland National Nature Reserve (ELWNNR) ( $44^{\circ} 50^{\prime} \mathrm{N}$, $\left.82^{\circ} 50^{\prime} \mathrm{E}\right)$ near the China-Kazakhstan border. It occupies an area of $\sim 650 \mathrm{~km}^{2}$, with a $2-4 \mathrm{~m}$ water depth, and the average altitude is over $189 \mathrm{~m}$. Inside the reserve, its ecology is almost undisturbed and has a complex biome structure and diverse habitats, such as small water bodies, swamps, and meadows. At present, there are hundreds of bird species, and numerous wild animals and plants have been found in this reserve, which has become an important component of China's biodiversity and a natural gene pool (Ma et al., 2010). In addition, Ebinur Lake is used as the main staging habitat during the migration of birds in Central Asia, where at least 200,000 water birds reside annually in the autumn (Ma et al., 2010). Hence, it provides a conducive habitat and suitable ecosystem for mosquito breeding and a virus-vectorhost cycle. In recent years, the China-Kazakhstan border has become a crucial region that connects China with countries in Central Asia and Europe. As a result, trade and travel across this region have rapidly increased, thus increasing the potential risk of the introduction and exportation of vectors and related diseases (The Lancet Global Health, 2017; Chen et al., 2019). However, at present, little is known regarding arboviruses associated with mosquitoes in the Ebinur Lake Wetland National Nature Reserve.

The family Peribunyaviridae was created to include the established bunyaviral genera Orthobunyavirus, Herbevirus, and Pacuvirus and the newly defined genus, Shangavirus (Abudurexiti et al., 2019). Currently, Orthobunyavirus is the largest genus within the Peribunyaviridae family and is distributed worldwide; it has a trisegmented negative-sense RNA genome comprising small (S), medium (M), and large (L) segments (Elliott, 2014). Reassortment and recombination are two important mechanisms in segmented RNA viruses, since these processes expand their viral genetic diversity and may significantly alter the virulence or other related biological properties (Gentsch et al., 1979; Lukashev, 2005; Briese et al., 2006, 2013; Shi et al., 2017; Nunes et al., 2019). Most orthobunyaviruses can infect vertebrates and exist in a zoonotic infection cycle, in which the transmission between humans and animals occurs via an arthropod intermediate. Human infections caused by viruses from the genus Orthobunyavirus can result in acute, mild febrile illnesses (Bunyamwera virus) to encephalitis (California encephalitis virus, La Crosse virus, or Tahyna virus) and hemorrhagic fevers (Ngari virus). In the past decade, novel orthobunyaviruses associated with human and/or livestock diseases (Iquitos virus, Itaya virus, Ntwetwe virus, and Schmallenberg virus) have been identified. Furthermore, known orthobunyaviruses have re-emerged or expanded to new geographical territories, a situation that has presented a serious economic and public health threat (Aguilar et al., 2011; Wernike and Beer, 2017; Edridge et al., 2018).

From our previously conducted mosquito-borne virus surveillance study in the Ebinur Lake region in 2014, a novel orthobunyavirus, Ebinur Lake virus (EBIV), which was previously named Abbey Lake virus, was isolated from Culex modestus mosquito pools, and its whole genome sequences were reported (Liu et al., 2014b,c). However, its detailed characterization and impact on animal or human health have not been investigated. Therefore, in the present study, we aimed to (1) extensively conduct EBIV's molecular analysis and outline its phylogenetic classification, (2) determine its in vitro infectivity range on various cell lines and in vivo infectivity pattern through the use of a mouse model, and (3) conduct an EBIV human seroprevalence study in the Ebinur Lake region. Certainly, our findings provide and subsequently expand the much-needed knowledge on EBIV, and this information can be utilized in the prevention and control of this neglected potential zoonotic virus.

\section{MATERIALS AND METHODS}

\section{Ethics Statement}

Animal studies were approved by the Animal Care and Use Committee of the Center for Disease Control and Prevention of Xinjiang Military Command Region (Approval No. 2014001). Human serum collection was approved by the Ethical Committee of the Center for Disease Control and Prevention of Xinjiang Military Command Region (Approval No. 2014006). All adult subjects provided informed consent, which was given in writing, and a parent or guardian of any child participant provided informed consent on the child's behalf.

\section{Cell Lines, Virus Stocks, and Animals}

Aedes albopictus C6/36 cell lines were grown in Roswell Park Memorial Institute 1640 medium with 1\% penicillin/streptomycin and $10 \%$ fetal bovine serum at $28^{\circ} \mathrm{C}$. BHK-21, Vero, and SW13 cells were grown in Dulbecco's modified Eagle's medium with $1 \%$ penicillin/streptomycin and $5-10 \%$ fetal bovine serum (Gibco, United States) at $37^{\circ} \mathrm{C}$ in $5 \% \mathrm{CO}_{2}$.

The EBIV isolate $\mathrm{Cu}-\mathrm{XJ} 20$ was originally isolated from C. modestus mosquitoes in 2013 in Xinjiang, China, by inoculation in suckling mice and passage three times in BHK-21 cells as a seed stock. Then, the working stock was generated, and the titer was $1.2 \times 10^{7}$ plaque-forming units $/ \mathrm{ml}(\mathrm{PFU} / \mathrm{ml})$.

Kunming mice, progeny of Swiss mice that are regarded as a viral infection animal model (Yu et al., 2017), were used in this study. Mice aged 6-8 weeks (20-25 g) were used since they have potent immune responses at this point. The mice were acquired from the Animal Center of Xinjiang Medical University and maintained in an ABSL-2 facility with controlled temperature $\left(22^{\circ} \mathrm{C}\right)$, humidity, and a 12 -h light/dark cycle.

\section{Seroprevalence Study Sites and the Collection of Serum Samples}

Alashankou and the Fifth Division of Xinjiang Production and Construction Corps were selected as human seroprevalence study sites. These two study sites are approximately 39 and $21 \mathrm{~km}$, respectively, from the site where EBIV was originally isolated from mosquitoes (Figure 1).

Between 2014 and 2015, a total of 211 human serum samples were collected from residents who visited the local outpatient 


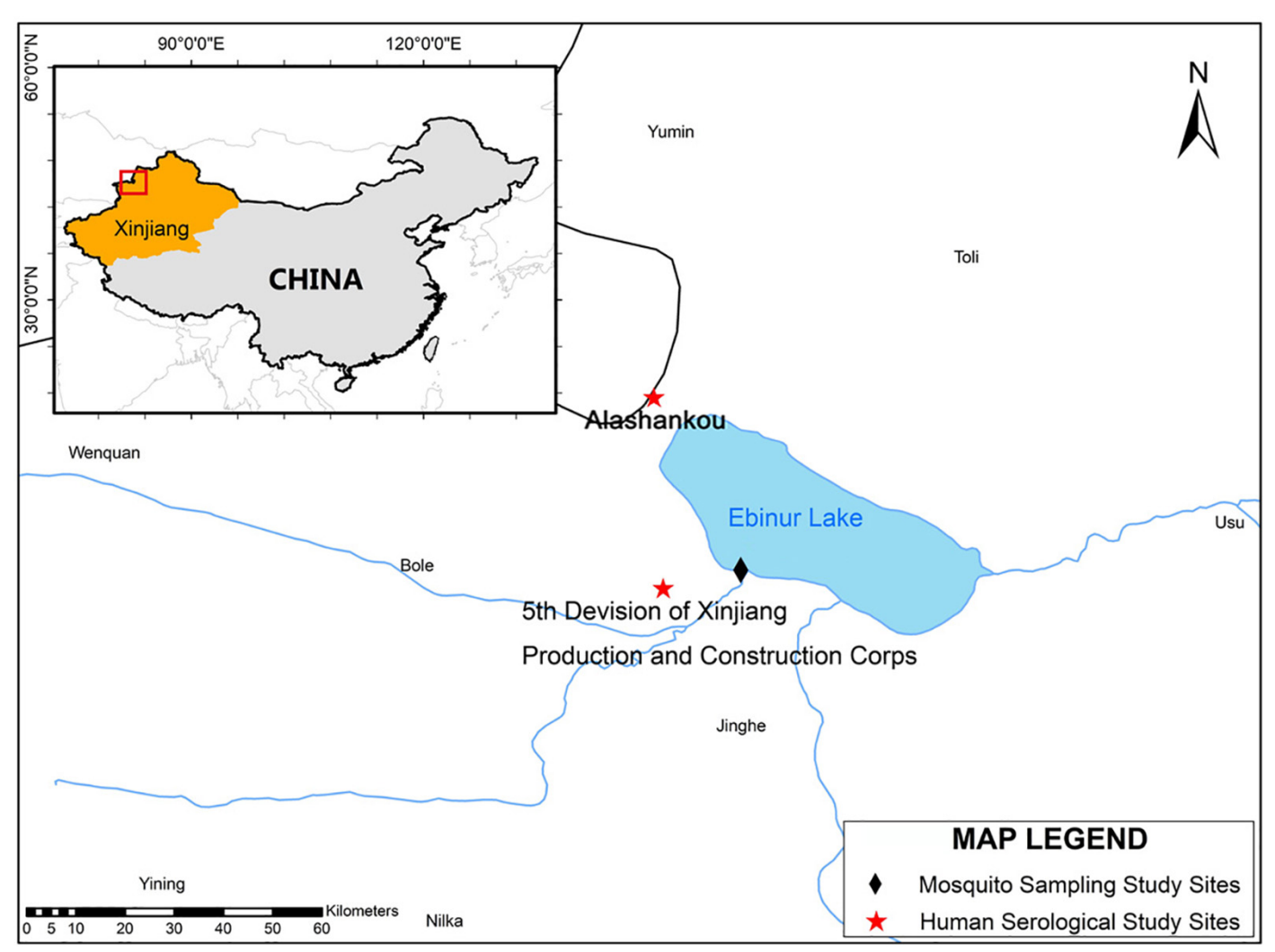

FIGURE 1 | The mosquito collection and serological study sites map. The map and the associated shapefiles were constructed and obtained from the free and open source Quantum GIS software (https://qgis.org/en/site/) and DIVA-GIS platform (https://www.diva-gis.org/gdata).

clinic during the high breeding season of mosquitoes. Study participants were divided into two groups: one group comprised individuals with no clinical symptoms visiting regular physical examinations, while the other group comprised individuals with fever $\left(>37.5^{\circ} \mathrm{C}\right)$ of unknown origin. The samples were collected within 1-3 days of the onset of clinical symptoms. Sociodemographic characteristics of the study population, including gender, age, location, and occupation, were collected from every study participant.

\section{Bioinformatics Analyses}

The complete coding sequences (CDSs) of all 3 segments of 55 strains (except the Germiston virus, which lacks $\mathrm{L}$ segment information) belonging to the serogroups of Bunyamwera, California, Group C, Guma, Mapputta, Patois, and Simbu in the Orthobunyavirus genus together with the representative members of Herbevirus (Herbert virus) were used in the analysis (from GenBank as of 28 December 2018). Background information of all the utilized strains is listed in Supplementary Table 1.

The nucleotide and amino acid sequence identities of the CDSs of EBIV and other orthobunyaviruses were analyzed using SDT1.2 ${ }^{1}$. Alignment of EBIV with the representative members

${ }^{1}$ http://web.cbio.uct.ac.za/ brejnev/ of the Peribunyaviridae family was conducted by the ClustalW function in MEGAv7.0.21 $1^{2}$. The GTR + I + G substitution model was selected as the best-fit nucleotide substitution model by jModelTest $2^{3}$. Phylogenetic analyses among nucleotide sequences were determined by the maximum likelihood method using the general time-reversible model with 1,000 bootstrap replicates in MEGA.

The phylogenetic trees and the percentage identities were evaluated to identify possible reassortment. The recombination Detection Program-4 (RDP4 v. 4.74$)^{4}$ was used to rule out intrasegment recombination.

\section{Electron Microscopy}

Viral particle purification was performed as previously described (Wang et al., 2017; Xia et al., 2018a). Electron microscopy imaging was performed using the negative contrast method. Pure virus particles were added to a Formvar carbon-coated copper grid for $10 \mathrm{~min}$ and negatively stained for $2-3 \mathrm{~min}$ with $2 \%$ phosphotungstic acid with the $\mathrm{pH}$ adjusted to 6.8 with $1 \mathrm{M} \mathrm{KOH}$. Then, the samples were examined with a Hitachi U8010 electron microscope (Japan).

\footnotetext{
${ }^{2}$ https://www.megasoftware.net/

${ }^{3}$ https://github.com/ddarriba/jmodeltest2

${ }^{4}$ http://web.cbio.uct.ac.za/ darren/rdp.html
} 


\section{Plaque Assay}

Prepared aliquots of 10-fold serial dilutions of EBIV in Dulbecco's modified Eagle's medium were inoculated onto the BHK-21 cell monolayers in 24-well plates for $1 \mathrm{~h}$. The cells were then covered with an overlay medium including $1.5 \%$ methylcellulose and incubated at $37^{\circ} \mathrm{C}$ for $3-4$ days to allow plaque development. Afterward, the infected cells were stained with $2 \%$ crystal violet in $30 \%$ methanol for $5 \mathrm{~min}$ at room temperature. The plaques were manually counted and measured.

\section{Growth Curves of EBIV in Cultured Cells}

C6/36, BHK-21, Vero, and SW13 cells grown in T75 flask plates were infected with EBIV at the multiplicity of infection (MOI) of $0.0001,0.01$, and 1 . After inoculation, $2 \mathrm{ml}$ supernatant was collected daily (day 1-7) from the T75 flask and then replenished with $2 \mathrm{ml}$ fresh medium. Viral titers of the collected samples were detected via plaque assay with BHK-21 cells. This experiment was repeated three times.

\section{Mice Challenge and Pathogenesis Experiments}

For the survival experiment, three groups of Kunming mice (five per group) were inoculated intraperitoneally (i.p.) with EBIV at $10^{6}, 10^{4}$, and $10^{2} \mathrm{PFU}(200 \mu \mathrm{l}$ per mouse), and one group (three per group) was mock infected. The experiment was repeated three times. The infected mice were observed daily until death, and during this observation period, their clinical symptoms and weight changes were recorded. For pathogenesis experiments, six mice were inoculated with EBIV at $10^{3} \mathrm{PFU}$ through the i.p. route. At the point of death, the mice were anesthetized, and the blood, brain, liver, kidney, spleen, and intestine were collected for virus titration and pathological examination. To determine viral titers in tissues, $1 \mathrm{mg}$ of tissue was removed during necropsy, homogenized in $200 \mu \mathrm{l}$ of phosphate-buffered saline (PBS), and then used in RNA extraction. Tissues used for pathological examination were immediately fixed with $10 \%$ paraformaldehyde for 16-24 $\mathrm{h}$ and embedded in paraffin, where they were processed and sectioned before staining with hematoxylin and eosin (H\&E). Thereafter, the obtained sections of EBIV-infected and mock-infected samples were viewed by light microscopy to analyze the histopathological changes. This was performed at the Department of Pathology, Center for Disease Control and Prevention in Xinjiang.

\section{Immunofluorescence Assay}

Vero cells infected with EBIV were cultured for 4 days and then harvested by trypsinization before being washed with PBS. The cells were spotted onto 14-well HT-coated glass slides and fixed with acetone at room temperature for $5 \mathrm{~min}$, and the slides were then stored at $-80^{\circ} \mathrm{C}$ until use. Serum samples were diluted with PBS to 1:4 and 1:10. The diluted samples were placed on EBIV slides and incubated under humidified conditions at $37^{\circ} \mathrm{C}$ for $1 \mathrm{~h}$. Next, the slides were washed three times with $0.01 \mathrm{M}$ PBS ( $\mathrm{pH}$ 7.4). Fluorescein isothiocyanate-conjugated goat antihuman immunoglobulin $\mathrm{G}(\mathrm{IgG})$ or immunoglobulin M (IgM) antibody was then added $(200 \mu \mathrm{l} /$ well), and the slides were incubated at $37^{\circ} \mathrm{C}$ for $30 \mathrm{~min}$ and rinsed with $\mathrm{PBS}$ to remove excess secondary antibody. The slides were visualized using a fluorescence microscope (Olympus, Japan). Normal human serum purchased from ImumunoReagents Inc. was also tested as the negative control under the same conditions in each immunofluorescence assay (IFA) test for verification.

\section{Neutralizing Antibody Test}

Immunofluorescence assay-positive samples were each tested using a plaque reduction neutralization test (PRNT) assay for EBIV. Briefly, sera were heat inactivated at $56^{\circ} \mathrm{C}$ for $30 \mathrm{~min}$ and diluted to 1:4, followed by twofold serial dilutions. Sera were then mixed with $100 \mathrm{PFU}$ of virus and incubated at $37^{\circ} \mathrm{C}$ for $1 \mathrm{~h}$. The virus-serum dilution mixtures were then inoculated into BHK-21 cell monolayers in 24-well plates for $1 \mathrm{~h}$ before adding an overlay. After 3 days of incubation at $37^{\circ} \mathrm{C}$, the plates were stained, and plaques were counted. All positive samples were further titrated to determine endpoint titers. The PRNT titer was calculated based on a 90\% reduction in plaque count (PRNT90).

\section{RNA Extraction and Quantitative Real-Time RT-PCR}

To determine the viral RNA in mouse blood or tissue, $20 \mu \mathrm{l}$ serum (separated from $100 \mu \mathrm{l}$ blood) with $130 \mu \mathrm{l}$ PBS or $150 \mu \mathrm{l}$ tissue homogenization supernatant was mixed with $450 \mu \mathrm{l}$ TRI reagent. RNA was extracted using Direct-zol RNA MiniPrep (Zymo Research, United States) following the manufacturer's instructions. The $S$ segment was cloned into the plasmid vector pBlunt with a T7 promoter (Wuhan Tianyi Huiyuan Bioscience \& Technology Inc., China) and then linearized by restriction enzyme digestion as a template for in vitro RNA transcription using the HiScribe ${ }^{\mathrm{TM}}$ T7 Quick High-Yield RNA Synthesis Kit (NEB, United States) according to the manufacturer's instructions. The transcripts were purified using the RNeasy Plus Mini Kit (Qiagen, Germany) and resuspended in $40 \mu \mathrm{l}$ of RNase-free water. The concentrations of in vitro transcribed RNAs were determined using a NanoDrop ND-2000 (Invitrogen, United States) and used as the RNA standard.

Ebinur Lake virus-specific quantitative real-time reverse transcription PCR (RT-PCR) was performed using the primers and probes targeting the $S$ segment as follows: probe $\left(5^{\prime}-\right.$ FAM-TTTTGGGTCCATCTCTTTCCTCTGC-TAMRA-3') and primers (forward: 5'-GGTACCTCTGGCGCATTGTCTTTTC$3^{\prime}$ and reverse: $5^{\prime}$-GAAAAATGGCATCACCTGGGAAAGT- $3^{\prime}$ ). The reaction was performed on a LightCycler v2.5 platform (Roche, Switzerland) using a PrimeScript RT-PCR Kit (Takara, China) in accordance with the manufacturer's instructions.

\section{Statistical Analyses}

All obtained statistical data were analyzed with R (version 3.5.1) ${ }^{5}$. The average survival time, percentage mortality, and KaplanMeier survival curves were obtained using the "survival" and "KMsurv" packages. One-way ANOVA was used to determine significant differences among groups of highest viral titers in

${ }^{5}$ https://www.r-project.org/ 
different cell lines and viral RNA copies in blood and tissue of EBIV-challenged mice.

\section{RESULTS}

\section{Molecular Phylogeny, Reassortment, and Recombination Analysis}

The phylogenetic relationships among EBIV, other members in the selected serogroups of Orthobunyavirus (Bunyamwera, California, Group C, Guama, Mapputta, Patois, and Simbu serogroup), and Herbert virus (the outgroup) were analyzed based on all three segments, except the Germiston virus, which only has complete CDSs for the S and M segments in GenBank.

The phylogenetic results of all three segments demonstrate that EBIV belongs to the genus Orthobunyavirus and clusters with the members of the Bunyamwera serogroup (Figures 2A-C). The nucleotide identity analysis matrix, based on the selected viruses, indicates that the $\mathrm{S}$ segment has the highest identity, whereas the $\mathrm{M}$ segment has the lowest identity within the Bunyamwera serogroup. The S, $\mathrm{M}$, and $\mathrm{L}$ segments of EBIV share the highest sequence similarity with those of Germiston virus (90.7\%), Germiston virus (77.3\%), and Bunyamwera virus (72.7\%), respectively (Figures 2D-F). The amino acid similarity analysis indicated that the amino acid sequences of the NP, GP, and L proteins of EBIV have the highest similarity with those of Germiston virus (95.7\%), Germiston virus (89.0\%), and Bunyamwera virus (80.3\%), respectively.

The overall topology of the obtained phylogenetic trees with the $\mathrm{S}$ and $\mathrm{L}$ segment sequences was similar, as EBIV clustered with the Germiston virus or alone formed an independent clade separate from the Bunyamwera virus-clade (highlighted in a light purple color in Figures 2A,C) members. However, for the $\mathrm{M}$ segment, EBIV clustered with Germiston virus, which is located in the Bunyamwera virus clade (highlighted in a light purple color in Figure 2B), indicating that EBIV is a potential reassortant virus.

In addition, analysis of the three segments of EBIV and those of other members of the Bunyamwera serogroup revealed a recombination event involving EBIV, Birao virus, and Northway virus in the $M$ segment (Supplementary Figure 1). No recombination was detected in the $\mathrm{S}$ or $\mathrm{L}$ segment. The recombination event was predicted using different algorithms (RDP, Bootscan, MaxChi, Chimaera) with a significance level set at $P \leq 0.05$ (Supplementary Table 2).

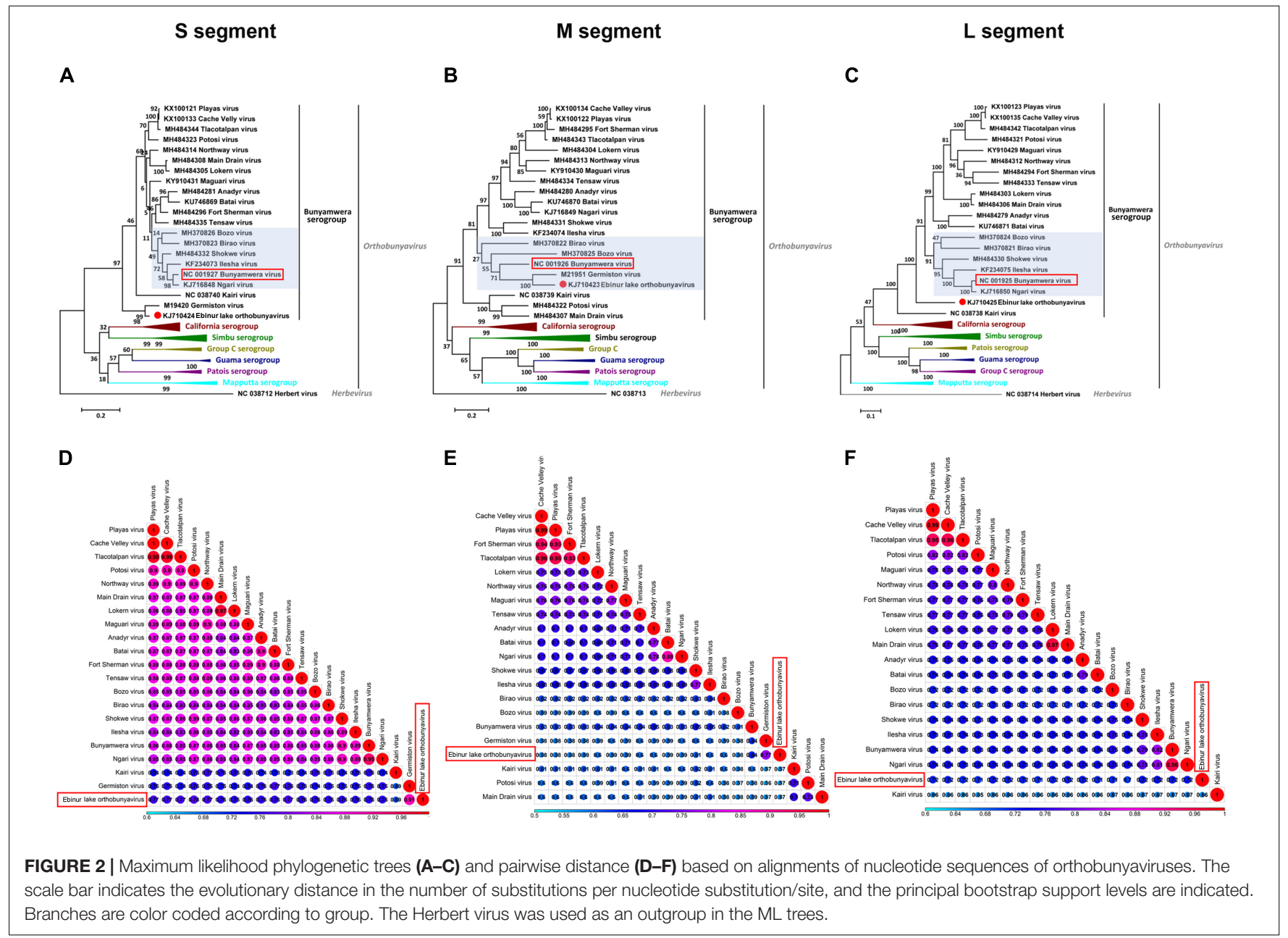




\section{Viral Morphology and Efficient Growth of EBIV in Mosquito and Mammalian Cell Lines}

Mature spherical, enveloped virions of approximately 90 $100 \mathrm{~nm}$ in diameter were detected in virus pellets generated by ultracentrifugation of EBIV-infected BHK-21 cell culture supernatants (Figure 3a), which was structurally similar to other members of the Orthobunyavirus genus. EBIV produced clear, ragged plaques (2-3 $\mathrm{mm}$ diameter) that were visible on the third day after inoculation in BHK-21 cell monolayers (Figure 3b).

To gain insight into the putative host tropism, the growth of EBIV was investigated using three different mammalian cell lines derived from hamster, monkey, or human (BHK, Vero, and SW13). Prominent cytopathic effects (CPEs) were observed in all cells, which were characterized by cell shrinking, shedding, and detachment from the growth surface from days 2 to 3 (Supplementary Figure 2). The virus replicated efficiently in these cells, and the growth kinetics demonstrated that virus titers reached a peak at $10^{8} \mathrm{PFU} / \mathrm{ml}$ in $\mathrm{BHK}$ (at $48 \mathrm{~h}$ when $\mathrm{MOI}=0.01$, at $72 \mathrm{~h}$ when MOI $=0.0001$ ) or in SW13 (at $48 \mathrm{~h}$ when MOI $=1$, at $72 \mathrm{~h}$ when MOI $=0.01)$ and then drastically decreased. In Vero cells, the highest virus titers $\left(10^{7} \mathrm{PFU} / \mathrm{ml}\right)$ were observed at $24 \mathrm{~h}$ with MOI $=1$ or at $72 \mathrm{~h}$ with MOI $=0.01$ and 0.0001 . In contrast, EBIV replicated much slower in mosquito C6/36 cells, which reached $10^{7} \mathrm{PFU} / \mathrm{ml}$ at $120 \mathrm{~h}$ with $\mathrm{MOI}=1$, and cells exhibited very slight changes. With MOI $=0.0001$, no virus could be detected between days 1 and 5 postinoculation. The significant difference $(P<0.01)$ for the highest virus titers was only observed between $\mathrm{C} 6 / 36$ and BHK cells (Figure 4).

\section{Mice Are Highly Susceptible to EBIV Infection}

All mice inoculated with $10^{6}$ and $10^{4}$ PFU of EBIV per animal died within 7 days. Twenty percent of the mice in the $10^{2} \mathrm{PFU}$ EBIV-inoculated group survived during the observation period (Figure 5A). The median survival time for the challenged mice was 4,4 , and 5 days in the groups treated with $10^{6}, 10^{4}$, and $10^{2}$ PFU, respectively. The infected mice exhibited clinical signs of illness manifested as decreased appetite, weight loss, ruffled fur, and general weakness. The viral RNAs in the blood and tissues of the infected mice were measured using quantitative RT-PCR. The virus could replicate in the blood and all other analyzed tissues. In addition, the brain tissue had the highest level of viral RNAs, with $\sim 10^{6}$ copies $/ \mathrm{mg}$, which presented a significant difference $(P<0.001)$ when compared to the other tested tissues, such as liver, spleen, and kidney (Figure 5B).

During necropsy, ascites was observed in the abdominal cavities of EBIV-challenged mice. In addition, the liver appeared discolored, and the intestines harbored dark contents, indicating internal bleeding (Figure 5C), whereas the organs of the mockinfected mice appeared normal.

To investigate the histopathological features of the EBIVinfected mice, the organs were harvested from infected mice. Prominent histopathological changes were observed in the liver, kidney, lung, and small intestine (Figure 6). The liver sections showed multiple foci of hepatocellular edema, and irregular vacuoles were observed in the cells. Histopathological changes in the small intestine consisted of necrosis of the intestinal mucosa, disappearance of intestinal glands, and destruction of tissue structure accompanied by massive hemorrhage. In addition, a large number of red blood cells were observed in the tubulointerstitial and alveolar tissues, and renal tubular protein casts could be observed. However, for the brain section, the vasodilatation and congestion of the blood vessel around meninges has been found, but no significant damage for brain neuron cells.

\section{Human Seroprevalence Study for EBIV}

The average age of the 211 participants was 36.9 years (standard deviation $=22.4$ ). The male/female ratio was $1.45: 1$. The majority of the study participants were farmers and $38.4 \%(81 / 211)$ were factory workers, followed by $22.3 \%$ (47/211) soldiers, $20.4 \%$ (43/211) children and students, and 18.9\% (40/211) retirees. Detailed characteristics of the tested sera are presented in Table 1.

Of the 211 tested serum samples, 17 were identified as IgM positive (1:4), 26 as IgG positive (1:10), and 4 as both IgM and IgG positive for EBIV by IFA (Supplementary Figure 3). In the participant with fever, higher IgM $(10.37 \%$ vs. 0$)$ and IgG (14.63 vs. $4.26 \%)$ positive rates were observed. Female

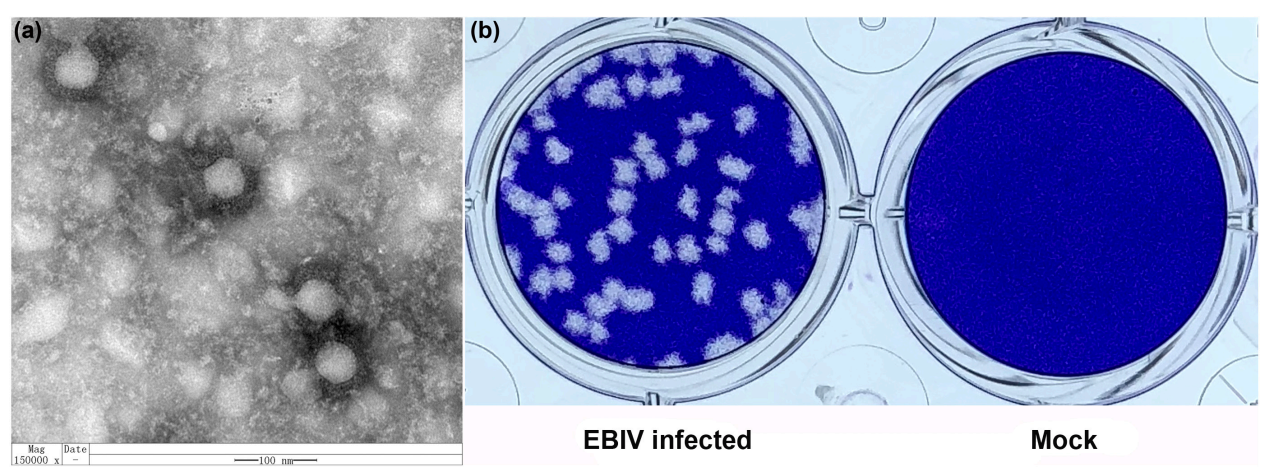

FIGURE 3 | The morphology and viral plaque in cells. (a) Negative-stained ultracentrifuged virions of EBIV. (b) Crystal violet-stained BHK-21 cell monolayers showing plaques generated by EBIV at $72 \mathrm{~h}$ postinfection. 


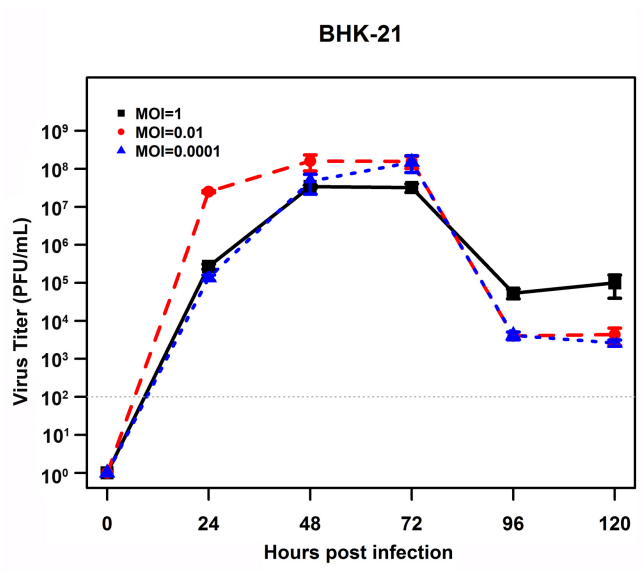

SW13

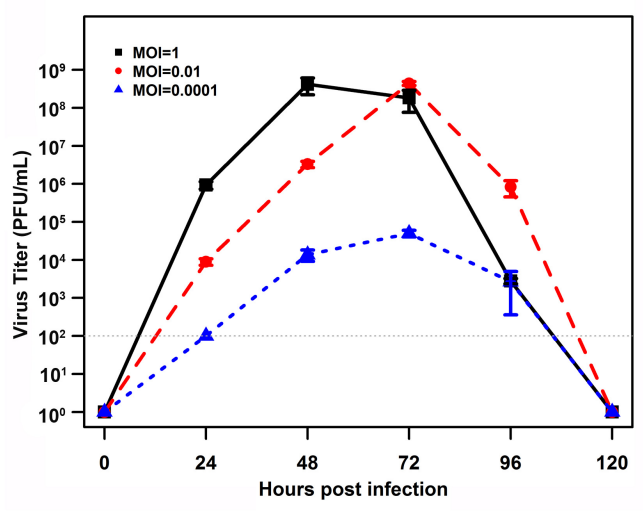

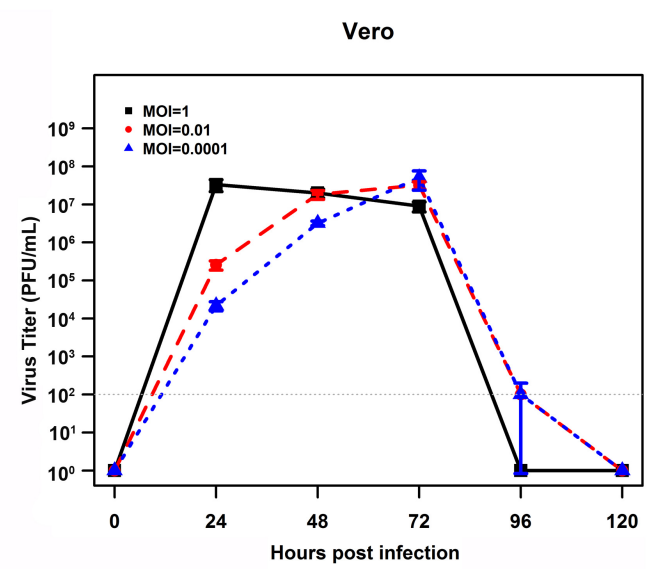

C6/36

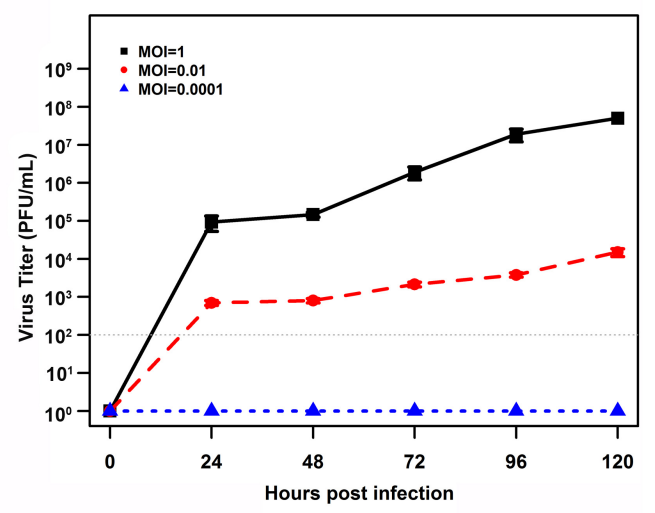

FIGURE 4 | Growth curves of EBIV (MOI = 1, 0.01, and 0.0001) in cells derived from mammals and mosquitoes. A significant difference in the highest virus titers was only observed between $\mathrm{C} 6 / 36$ and BHK cells (C6/36 vs. BHK with $P<0.01$, SW13 vs. BHK with $P=0.02$, Vero vs. BHK with $P=0.03$, SW13 vs. Vero with $P=0.99$, C6/36 vs. SW13 with $P=0.66$, and C6/36 vs. Vero with $P=0.55)$.

participants had a much higher IgM-positive rate than male participants (13.95 vs. $4.00 \%$ ), but no obvious difference was observed for IgG (13.95 vs. 9.6\%). Study participants belonging to the $>60$-year age group had the highest positive rate of both IgM (16.67\%) and IgG (12.69\%) among all age groups. Furthermore, based on the occupational groups, the proportion of positive samples for IgM and IgG was highest among retirees (20\%), followed closely by farmers and factory workers (17.28\%). In addition, the neutralizing antibody prevalence of EBIV was $0.95 \%$ $(2 / 211)$ (Table 1). For the two neutralizing antibody-positive cases, one male participant aged 50 years had a 1:8 PRNT90 titer, and the second case was from a female participant aged 50 years with a 1:16 PRNT90 titer. Both of these two study participants were from the Fifth Division of Xinjiang Production and Construction Corps region.

\section{DISCUSSION}

The present study further characterized EBIV, which was previously isolated from C. modestus mosquitoes collected from the Ebinur Lake region. The EBIV segments $S$ and $\mathrm{M}$ showed considerable genetic distance to currently known viruses, with 11 and $19 \%$ distance from the NP and G proteins of its closest relative, GERV. In contrast to dengue virus, Japanese encephalitis virus and other mosquito-borne flaviviruses, orthobunyaviruses are quite underestimated with regard to their potential prevalence and distribution in mainland China. To the best of our knowledge, Batai virus from the Bunyamwera serogroup and Tahyna virus from the California encephalitis serogroup are the only two orthobunyaviruses that have been documented in China (Lu et al., 2009; Liu et al., 2011, 2014a; Xia et al., 2018b). Hence, describing a newly identified Orthobunyavirus member is beneficial in understanding the diversity of orthobunyaviruses in China.

The genetic reassortment of viruses with triplicate segments in the order Bunyavirales plays an important role in driving the diversity and evolution of this virus group. For example, Nigari virus, which was characterized as a progeny from parental Batai virus and Bunyamwera virus, was implicated in hemorrhagic fever outbreaks in East Africa (Briese et al., 2006). In addition, natural $\mathrm{M}$ segment reassortment has been observed in Potosi 


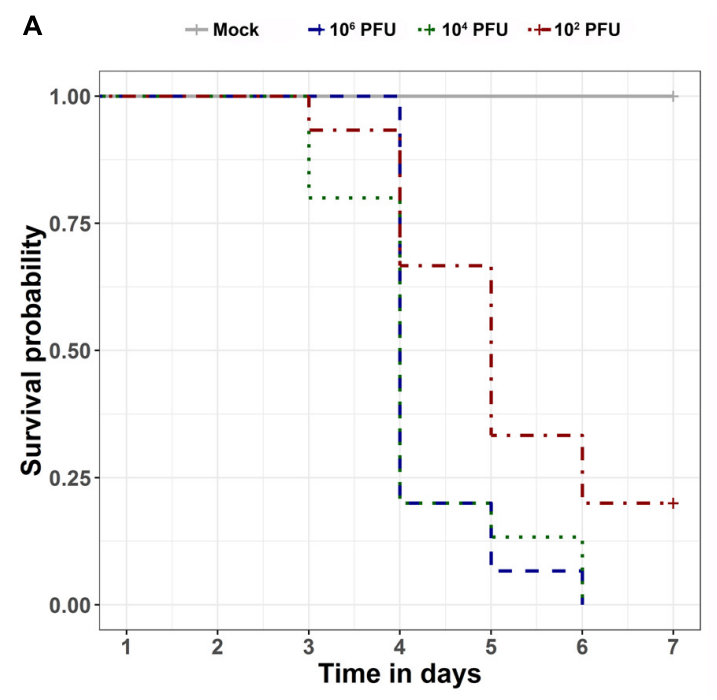

c EBIV-infected

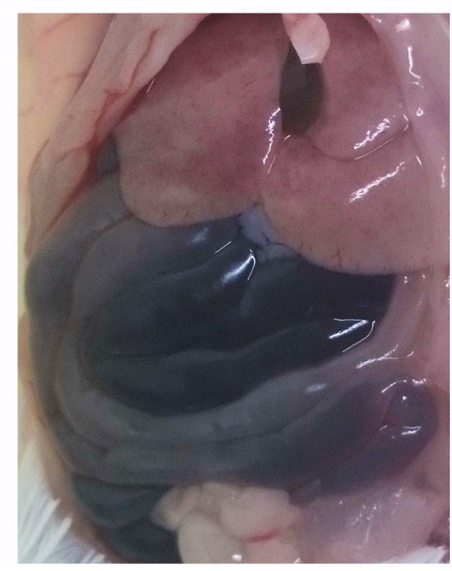

B

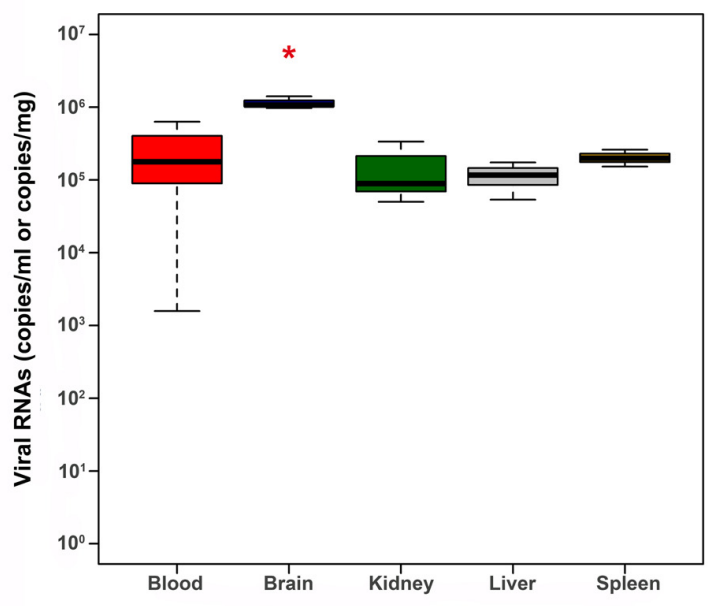

Mock

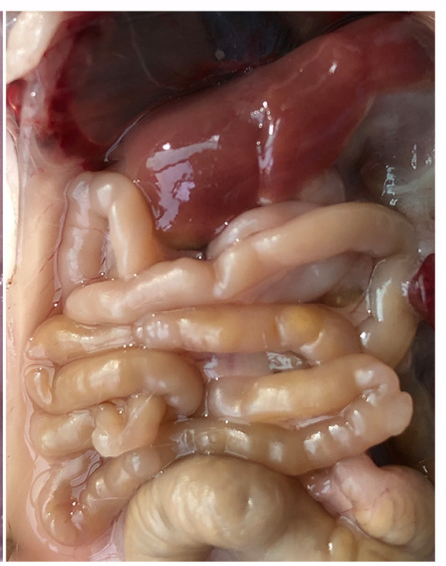

FIGURE 5 | Survival analysis, viral RNA detection in tissues, and gross pathology findings of EBIV-challenged mice. (A) Survival curve of mice challenged with serial dilutions of EBIV. Adult mice ( $n=5$ per group, replicated three times) were challenged intraperitoneally with $10^{6} \mathrm{PFU}$ (dark blue), $10^{4} \mathrm{PFU}$ (dark green), and $10^{2}$ PFU (dark red) of EBIV. (B) The viral RNA copies in blood, brain, kidney, liver, and spleen tissues were detected by quantitative RT-PCR, and the brain tissue presented a significant difference $\left({ }^{\star} P<0.05\right)$ compared to the other tested tissues. (C) In situ picture of organs in EBIV-infected animals with hemorrhaging and mock-infected animals.

and Main Drain viruses (Briese et al., 2007). In the present study, a potential reassortment signal of the $M$ segment was observed through the different phylogenetic tree topologies of the $M$ segment sequences compared to the $S$ and $L$ segment sequences. Moreover, a small $M$ segment recombination event was observed between EBIV, Birao virus, and Northway virus, which are viruses identified from China, Africa, and North America, respectively. The long-distance spread of these viruses may be due to migratory birds. Because there are two migratory bird flyways (Central Asian flyway and West Asian-East African flyway) that pass or cover the region of Xinjiang (Olsen et al., 2006), the viruses could have been carried by birds from Africa or America to China, and then, the recombination event occurred in local invertebrate or vertebrate hosts. The other probable possibility is that Xinjiang spans over 1.6 million $\mathrm{km}^{2}$, with no systematic research and surveillance studies for mosquito-borne virus; hence, other orthobunyaviruses that are similar to the Northway or Birao virus might be circulating in Xinjiang and are currently not identified or underreported. The recombinant region is located at the beginning of Gc in the glycoprotein. The N-terminal domain of Gc of viruses in the Bunyamwera serogroup is not essential for the infection of and replication in cultured cells, but the N-terminal domain does play some role in the infection process (Shi et al., 2009). This recombination event may have enhanced or impaired the viral ability to infect natural hosts. However, due to the small quantity of available virus sequences used in the analysis and the low nucleotide identity between EBIV and other members in the Bunyamwera serogroup, much more work should be done to clearly provide evidence of the reassortment and recombination events in EBIV.

Genetic analysis of EBIV based on its $S$ and $M$ segments showed that it was closely related to GERV, a virus that 


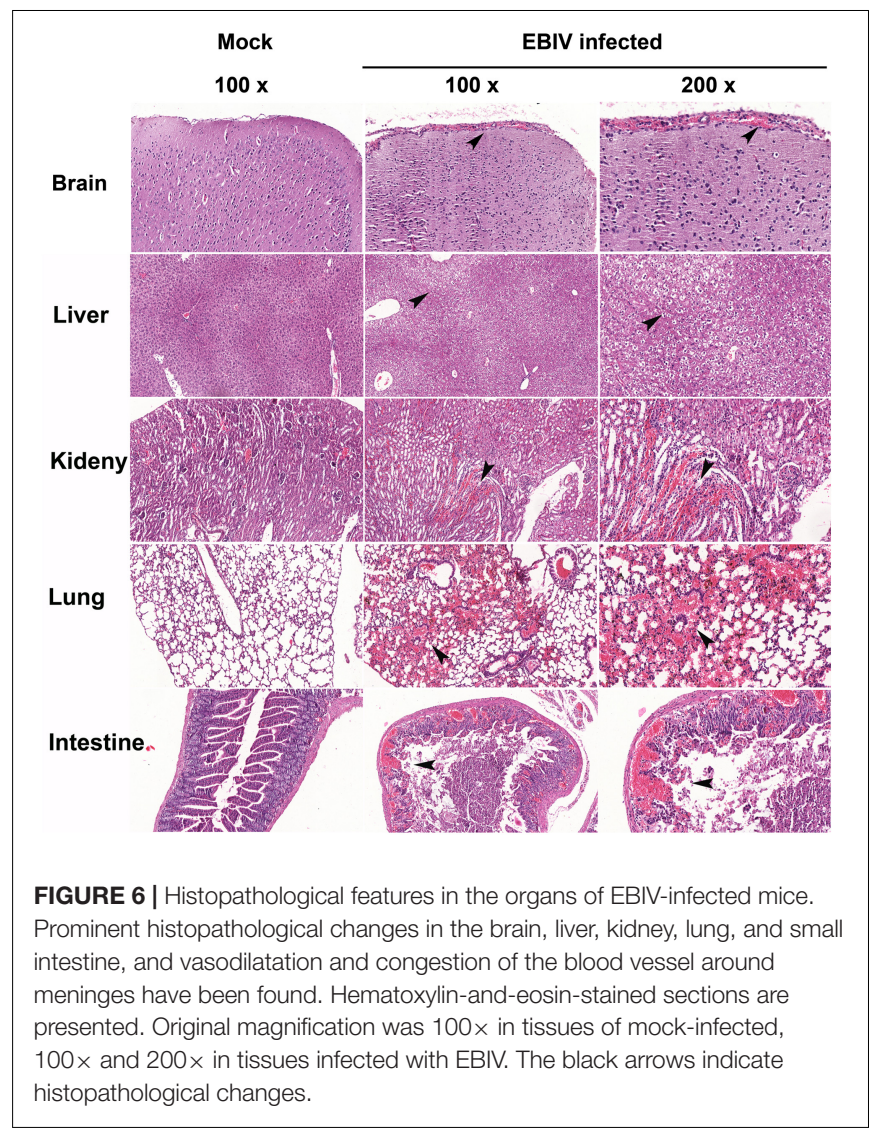

was isolated almost 50 years ago from Aedes circumluteolus mosquitoes and rodents in South Africa and is associated with human infections (Monath et al., 1972; Venter, 2018). From our findings, in vitro tests indicate that the virus grew faster and caused more obvious CPEs in mammalian cell lines while causing a persistent infection in mosquito cells without obvious CPEs. These findings are in agreement with other previously reported studies on members of the Bunyamwera serogroup. The viral NSs protein could be one of the factors responsible for the different infection outcomes of orthobunyaviruses in mammalian and mosquito cell lines (Szemiel et al., 2012). The in vivo infection experiment showed that EBIV causes a lethal infection in adult mice through i.p. route. This finding corresponds with the reported results on GERV, but detailed information on the pathology changes of mice to GERV infection is not available (Monath et al., 1972). There are reports indicating that several members of orthobunyaviruses are neurovirulence in lab experimental animals, such as mice and hamsters. High viral titers could be detected in the brain tissue of sick mice and exhibit paralysis and convulsion signs (Wilson et al., 2015). In this study, the significant CNS symptoms such as paralysis, ataxia, or convulsion and the damage for brain neuron cells has not been detected in EBIV-infected Kunming mice. Since the EBIV presenting 10-30\% genetic divergence with the other bunyamwera serogroup member, EBIV could have difference neurovirulence or neurotropism features in mice. Therefore, more in-depth studies should be conducted to fully
TABLE 1 | Study participant characteristics and human serum samples positive for EBIV.

\begin{tabular}{|c|c|c|c|}
\hline \multirow[t]{2}{*}{ Group } & \multicolumn{3}{|c|}{ Positive rate $(\%)$} \\
\hline & $\lg M 1: 4$ (IFA) & IgG 1:10 (IFA) & PRNT90 \\
\hline \multicolumn{4}{|l|}{ Gender } \\
\hline Male & $4.00(5 / 125)$ & $9.6(14 / 125)$ & $0.8(1 / 125)$ \\
\hline Female & $13.95(12 / 86)$ & $13.95(12 / 86)$ & $1.16(1 / 86)$ \\
\hline \multicolumn{4}{|l|}{ Age group (years) } \\
\hline $0-20$ & $9.52(4 / 52)$ & $11.9(6 / 52)$ & $0(0 / 52)$ \\
\hline $21-40$ & $4.34(1 / 60)$ & $9.52(5 / 60)$ & $0(0 / 60)$ \\
\hline $41-60$ & $9.52(6 / 63)$ & $11.11(7 / 63)$ & $3.17(2 / 63)$ \\
\hline$>60$ & $16.67(6 / 36)$ & $12.69(8 / 63)$ & $0(0 / 63)$ \\
\hline \multicolumn{4}{|l|}{ Occupation } \\
\hline Child and student & $9.3(4 / 43)$ & $11.63(5 / 43)$ & $0(0 / 43)$ \\
\hline Farmer and factory worker & $6.17(5 / 81)$ & $17.28(14 / 81)$ & $2.47(2 / 81)$ \\
\hline Soldier & $0(0 / 47)$ & $4.26(2 / 47)$ & $0(0 / 47)$ \\
\hline Retiree & $20(8 / 40)$ & $12.5(5 / 40)$ & $(0 / 40)$ \\
\hline \multicolumn{4}{|l|}{ With fever symptoms } \\
\hline No & $0(0 / 47)$ & $4.26(2 / 47)$ & $0(0 / 47)$ \\
\hline Yes & $10.37(17 / 164)$ & $14.63(24 / 164)$ & $1.22(2 / 164)$ \\
\hline Total & $8.05(17 / 211)$ & $12.3(26 / 211)^{\mathrm{a}}$ & $0.95(2 / 211)^{b}$ \\
\hline
\end{tabular}

a The IgM-positive population was not the same as the lgG-positive population; only four cases were both IgM and IgG positive. ${ }^{b}$ These two PRNT-positive patients were positive for IgG but not for IgM.

comprehend the pathogenesis and immune response of EBIV in different animal models.

In human seroepidemiological surveys, a cross-reaction could occur in the IFA or PRNT test, and no other confirmatory data, such as positive RT-PCR results, viral isolation, or later convalescent sera test results, were available; therefore, no clinical conclusions for this human infection for EBIV can be drawn. However, these results provide us with some insights into EBIV infection in local residents, providing new ideas for clinicians and researchers to diagnose unknown fever cases in this region. We will follow up on these results to determine whether EBIV could cause human cases in this region with the following measures: (1) neutralization test for patients with acute and convalescent sera to see if the neutralizing antibody titer is increased by four or more times; (2) virus isolation in serum samples from patients in the acute phase; and (3) nucleic acid detection of patient specimens in the acute phase. Furthermore, the presence of EBIV nucleic acids and antibodies in local birds and vertebrates will be conducted to help us understand the natural cycle of EBIV in this region.

The Xinjiang region is demarked as the main gateway of China's Silk Road economic corridor to Central Asia and Europe. As a result, this region is experiencing rapid growth in terms of infrastructure and human population development. Consequently, the deforestation rate has extensively increased in recent years. In turn, this scenario has augmented the risk for the emergence of zoonoses. Most importantly, the Ebinur Lake Nature Reserve is located at the China-Kazakhstan border, a region with rich biodiversity that provides an appropriate environment for the virus-mosquito-host cycle. Therefore, more surveillance and experimental transmission studies should be 
conducted in this region to determine the threat of this neglected EBIV to animal and human health.

\section{DATA AVAILABILITY STATEMENT}

Publicly available datasets were analyzed in this study. This data can be found here: KJ710424, KJ710423, KJ710425, MH484281, MH484280, MH484279, KU746869, KU746870, KU746871, MH370823, MH370822, MH370821, MH370826, MH370825, MH370824, NC_001927, NC_001926, NC_001925, KX100133, KX100134, KX100135, MH484296, MH484295, MH484294, M19420, M21951, KF234073, KF234074, KF234075, NC_038740, NC_038739, NC_038738, MH484305, MH484304, MH484303, KY910431, KY910430, KY910429, MH484308, MH484307, MH484306, KJ716848, KJ716849, KJ716850, MH484314, MH484313, MH484312, KX100121, KX100122, KX100123, MH484323, MH484322, MH484321, MH484332, MH484331, MH484330, MH484335, MH484334, MH484333, MH484344, MH484343, MH484342, KX817314, KX817313, KX817312, MG765471, MG765470, MG765469, KT288271, KT288270, KT288269, KX817335, KX817334, KX817333, NC_004110, NC_004109, NC_004108, KX817332, KX817331, KX817330, EU294510, EU262553, EU203678, KX817338, KX817337, KX817336, HM243139, HM243138, HM243137, KX891323, KX891322, KX891321, MG029274, MG029273, MG029272, NC_034498, NC_034505, NC_034497, MG029283, MG029282, MG029281, MG029292, MG029291, MG029290, NC_038725, NC_038723, NC_038724, NC_038726, NC_038727, NC_038728, NC_038737, NC_038735, NC_038736, KJ481927, KJ481928, KJ481929, KJ481921， KJ481922， KJ481923， NC_026282, NC_026283, NC_026281, NC_022597, NC_022596, NC_022595, MH017269, MH017281, MH017275, MH017270, MH017282, MH017276, MH017273, MH017283, МH017277, MH017271, MH017284, MH017278, NC_009896, NC_009895, NC_009894, JQ675601, JQ675602, JQ675603, MH484338, MH484337, MH484336, MF926352, MF926353, MF926354, NC_018462, NC_018466, NC_018461, NC_018464, NC_018467, NC_018463, NC_018460, NC_018459, NC_018465, NC_018477, NC_018478, NC_018476, NC_038712, NC_038713, and NC_038714.

\section{ETHICS STATEMENT}

The studies involving human participants were reviewed and approved by the Ethical Committee of the Center for Disease Control and Prevention of Xinjiang Military Command Region Approval No. 2014006. Written informed consent to participate

\section{REFERENCES}

Abudurexiti, A., Adkins, S., Alioto, D., Alkhovsky, S. V., Avšiè-Županc, T., Ballinger, M. J., et al. (2019). Taxonomy of the order Bunyavirales: update 2019. Arch. Virol. 164, 1949-1965. doi: 10.1007/s00705-019-04253-4256

Aguilar, P. V., Barrett, A. D., Saeed, M. F., Watts, D. M., Russell, K., Guevara, C., et al. (2011). Iquitos virus: a novel reassortant orthobunyavirus associated with human illness in peru. PLoS Negl. Trop. Dis. 5:e1315. doi: 10.1371/journal.pntd. 0001315 in this study was provided by the participants' legal guardian/next of kin. The animal study was reviewed and approved by the Animal Care and Use Committee of the Center for Disease Control and Prevention of Xinjiang Military Command Region Approval No. 2014001.

\section{AUTHOR CONTRIBUTIONS}

ZY, HX, and GZ designed the experiments. HX, RL, and LZ performed the experiments. HX, LZ, and EA analyzed the data. $\mathrm{ZY}, \mathrm{XS}, \mathrm{ZZ}$, and $\mathrm{XH}$ contributed the reagents, materials, and analysis tools. LZ, HX, EA, GZ, and ZY wrote the manuscript. $\mathrm{BZ}$ wrote and reviewed the manuscript.

\section{FUNDING}

This work was supported by the Ministry of Science and Technology of the People's Republic of China (2018ZX10101004 and 2013FY113500); the National Health Commission of the People's Republic of China (2018ZX10711001-006); the Chinese Academy of Sciences (153211KYSB20160001 and ZDRW-ZS2016-4); the Wuhan Institute of Virology, China (WIV-135-PY2); the Health Commission of Hubei Province (WJ2019Q060); the National Natural Science Funds of China (U1303104); and the Chinese Academy of Military Medical Sciences (SKLPBS144).

\section{ACKNOWLEDGMENTS}

We would like to acknowledge Dr. Hong Liu (Shandong University of Technology) and Dr. Haizhou Liu (Wuhan Institute of Virology, Chinese Academy of Sciences) for critically reviewing this manuscript. Equally, we sincerely thank Pei Zhang and Anna Du from the Core Facility and Technical Support of Wuhan Institute of Virology for their help with producing TEM micrographs and Jianglin Yuan from the Department of Pathology, Center for Disease Control and Prevention in Xinjiang for help with pathology analysis.

\section{SUPPLEMENTARY MATERIAL}

The Supplementary Material for this article can be found online at: https://www.frontiersin.org/articles/10.3389/fmicb. 2019.03111/full\#supplementary-material

Briese, T., Bird, B., Kapoor, V., Nichol, S. T., and Lipkin, W. I. (2006). Batai and Ngari viruses: $\mathrm{m}$ segment reassortment and association with severe febrile disease outbreaks in East Africa. J. Virol. 80, 5627-5630. doi: 10.1128/JVI. 02448-2445

Briese, T., Calisher, C. H., and Higgs, S. (2013). Viruses of the family Bunyaviridae: are all available isolates reassortants? Virology 446, 207-216. doi: 10.1016/j.virol. 2013.07.030

Briese, T., Kapoor, V., and Lipkin, W. I. (2007). Natural M-segment reassortment in Potosi and Main Drain viruses: implications for the evolution of 
orthobunyaviruses. Arch. Virol. 152, 2237-2247. doi: 10.1007/s00705-0071069-z

Chen, J., Bergquist, R., Zhou, X.-N., Xue, J.-B., and Qian, M.-B. (2019). Combating infectious disease epidemics through China's Belt and Road Initiative. PLoS Negl. Trop. Dis. 13:e0007107. doi: 10.1371/journal.pntd.0007107

Edridge, A. W. D., Deijs, M., Namazzi, R., Cristella, C., Jebbink, M. F., Maurer, I., et al. (2018). Novel orthobunyavirus identified in the cerebrospinal fluid of a Ugandan child with severe encephalopathy. Clin. Infect. Dis. 68, 139-142. doi: $10.1093 / \mathrm{cid} / \mathrm{ciy} 486$

Elliott, R. M. (2014). Orthobunyaviruses: recent genetic and structural insights. Nat. Rev. Microbiol. 12, 673-685. doi: 10.1038/nrmicro3332

Gentsch, J. R., Robeson, G., and Bishop, D. H. (1979). Recombination between snowhoe hare and La Crosse bunyaviruses. J. Virol. 31, 707-717. doi: 10.1128/ jvi.31.3.707-717.1979

Hollidge, B. S., González-Scarano, F., and Soldan, S. S. (2010). Arboviral encephalitides: transmission, emergence, and pathogenesis. J. Neuroimmune Pharmacol. 5, 428-442. doi: 10.1007/s11481-010-9234-9237

Liu, H., Gao, X., and Liang, G. (2011). Newly recognized mosquito-associated viruses in mainland China, in the last two decades. Virol. J. 8:68. doi: 10.1186/ 1743-422X-8-68

Liu, H., Shao, X., Hu, B., Zhao, J., Zhang, L., Zhang, H., et al. (2014a). Isolation and complete nucleotide sequence of a Batai virus strain in Inner Mongolia. China. Virol. J. 11:138. doi: 10.1186/1743-422X-11-138

Liu, R., Zhang, G., Sun, X., Zheng, Z., Liu, X., Zhao, Y., et al. (2014b). Isolation and molecular characterization on Abbey Lake Orthobunyavirus (Bunyaviridae) in Xinjiang. China. Zhonghua Liu Xing Bing Xue Za Zhi 35, 939-942.

Liu, R., Zhang, G., Yang, Y., Dang, R., and Zhao, T. (2014c). Genome sequence of abbey lake virus, a novel orthobunyavirus isolated from China. Genome Announc. 2:e00433-14. doi: 10.1128/genomeA.00433-14

Lu, Z., Lu, X.-J., Fu, S. H., Zhang, S., Li, Z. X., Yao, X. H., et al. (2009). Tahyna virus and human infection China. Emerg. Infect. Dis. 15, 306-309. doi: 10.3201/ eid1502.080722

Lukashev, A. N. (2005). Evidence for recombination in Crimean-Congo hemorrhagic fever virus. J. Gen. Virol. 86(Pt 8), 2333-2338. doi: 10.1099/vir. 0.80974-80970

Ma, M., Bayahen, K., Li, F., Hu, B., Wu, J., Douglas, M., et al. (2010). List of birds and count of autumn migration in ebinur wetland nature reserve. Sichuan $J$. Zool. 29, 912-918.

Monath, T. P. C., Henderson, B. E., and Kirya, G. B. (1972). Characterization of viruses (Witwatersrand and Germiston) isolated from mosquitoes and rodents collected near Lunyo Forest, Uganda, in 1968. Arch. Gesamte Virusforsch. 38, 125-132. doi: 10.1007/BF01249661

Nunes, M. R. T., de Souza, W. M., Savji, N., Figueiredo, M. L., Cardoso, J. F., da Silva, S. P., et al. (2019). Oropouche orthobunyavirus: genetic characterization of full-length genomes and development of molecular methods to discriminate natural reassortments. Infect. Genet. Evol. 68, 16-22. doi: 10.1016/j.meegid. 2018.11.020
Olsen, B., Munster, V. J., Wallensten, A., Waldenström, J., Osterhaus, A. D., and Fouchier, R. A. M. (2006). Global patterns of influenza A virus in wild birds. Science 312, 384-388. doi: 10.1126/science.1122438

Shi, J., Hu, S., Liu, X., Yang, J., Liu, D., Wu, L., et al. (2017). Migration, recombination, and reassortment are involved in the evolution of severe fever with thrombocytopenia syndrome bunyavirus. Infect. Genet. Evol. 47, 109-117. doi: 10.1016/j.meegid.2016.11.015

Shi, X., Goli, J., Clark, G., Brauburger, K., and Elliott, R. M. (2009). Functional analysis of the Bunyamwera orthobunyavirus Gc glycoprotein. J. Gen. Virol. 90, 2483-2492. doi: 10.1099/vir.0.013540-13540

Szemiel, A. M., Failloux, A. B., and Elliott, R. M. (2012). Role of Bunyamwera orthobunyavirus NSs protein in infection of mosquito cells. PLoS Negl. Trop. Dis. 6:e1823. doi: 10.1371/journal.pntd.0001823

The Lancet Global Health. (2017). Facing forwards along the Health Silk Road. Lancet Glob. Heal. 5:e948. doi: 10.1016/S2214-109X(17)30349-30342

Venter, M. (2018). Assessing the zoonotic potential of arboviruses of African origin. Curr. Opin. Virol. 28, 74-84. doi: 10.1016/j.coviro.2017.11.004

Wang, Y., Xia, H., Zhang, B., Liu, X., and Yuan, Z. (2017). Isolation and characterization of a novel mesonivirus from Culex mosquitoes in China. Virus Res. 240, 130-139. doi: 10.1016/j.virusres.2017.08.001

Wernike, K., and Beer, M. (2017). schmallenberg virus: a novel virus of veterinary importance. Adv. Virus Res. 99, 39-60. doi: 10.1016/bs.aivir.2017.07.001

Wilson, W. C., Gaudreault, N. N., Hossain, M. M., and McVey, D. S. (2015). Lesserknown bunyavirus infections. OIE Rev. Sci. Tech. 34, 419-429. doi: 10.20506/rst. 34.2.2368

Xia, H., Liu, H., Zhao, L., Atoni, E., Wang, Y., and Yuan, Z. (2018a). First isolation and characterization of a group C Banna virus (BAV) from Anopheles sinensis Mosquitoes in Hubei China. Viruses 10:555. doi: 10.3390/v10100555

Xia, H., Wang, Y., Atoni, E., Zhang, B., and Yuan, Z. (2018b). Mosquito-Associated Viruses in China. Virol. Sin. 33, 5-20. doi: 10.1007/s12250-018-0002-9

Yu, J., Liu, X., Ke, C., Wu, Q., Lu, W., Qin, Z., et al. (2017). Effective suckling $\mathrm{C} 57 \mathrm{Bl} / 6$, kunming, and BALB/C mouse models with remarkable neurological manifestation for zika virus infection. Viruses 9:E165. doi: 10.3390/v9070165

Conflict of Interest: RL is employed by Illumina, China.

The remaining authors declare that the research was conducted in the absence of any commercial or financial relationships that could be construed as a potential conflict of interest.

Copyright (C) 2020 Xia, Liu, Zhao, Sun, Zheng, Atoni, Hu, Zhang, Zhang and Yuan. This is an open-access article distributed under the terms of the Creative Commons Attribution License (CC BY). The use, distribution or reproduction in other forums is permitted, provided the original author(s) and the copyright owner(s) are credited and that the original publication in this journal is cited, in accordance with accepted academic practice. No use, distribution or reproduction is permitted which does not comply with these terms. 